\title{
FREE VIBRATION ANALYSIS OF SIMPLY SUPPORTED P-FGM NANOPLATE USING A NONLOCAL FOUR VARIABLES SHEAR DEFORMATION PLATE THEORY
}

\author{
CHIKH Abdelbaki ${ }^{1}$ \\ ${ }^{I}$ Department of Civil Engineering, Faculty of Applied Sciences, Ibn Khaldoun University, Tiaret, Algeria \\ e - mail: cheikhabdelbakki@yahoo.fr
}

\begin{abstract}
This paper shows an analysis of the free vibration of functionally graded simply supported nanoplate. The nonlocal four variables shear deformation plate theory is used to predict the free vibration frequencies of functionally graded nanoplate simply supported using non-local elasticity theory with the introduction of smallscale effects. The effect of the material properties, thickness-length ratio, aspect ratio, the exponent of the power law, the vibration mode is presented, the current solutions are compared to those obtained by other researchers. Equilibrium equations are obtained using the virtual displacements principle. P-FGM Power law is used to have a distribution of material properties that vary across the thickness. The results are in good agreement with those of the literature.
\end{abstract}

KEYWORDS: FGM nanoplate; simply supported nanoplate; small-scale effects; nonlocal theory

\section{Introduction}

Functionally graded materials attract the attention of many researchers because of their powerful uses and their characteristics (mechanical, chemical, thermal, physical) such as high abrasion resistance (ceramic face), high impact resistance, reactor components, and insulating joints. Dimming improves the toughness of the ceramic face and prevents ceramic-metal detachment. Functionally graded materials (FGM) can be characterized by the gradual variation of material properties in the thickness. A new type of composite materials is developed recently (Abdelbaki et al [1]; Arnab Choudhury et al [2]; Abdelbaki et al [3]; Ebrahimi and Barati [4]; Ebrahimi and Heidari [5]; Elmerabet et al [6]; Elmossouess et al [7]; Houari et al [8]; Karami et al [9]; Mahjoobi and Bidgoli [10]; Mohamed et al [11]; Mokhtar et al [12]; Mokhtar et al [13]; Sadoun et al [14]; Salari et al [15]; Shafiei and Setoodeh [16]; Shokravi [17]; Tlidji et al [18]; Tounsi et al [19]; Tu et al [20]; Bocko, J et al [21]; Jozef, B et al [22]; Stephan, K et al [23]; Murín, J et al [24]; Sapountzakis, E et al [25]).

Research work dealing with the behavior of nanoplates under different types of loading can be cited as Ansari and Norouzzadeh [26] studied the buckling responses of circular, elliptical and asymmetric nanometric plates in FGM. Banh-Thien et al [27] presented a new numerical approach for the buckling analysis of non-uniform thick nanoplates in an elastic medium using isogeometric analysis (IGA). Ghadiri et al [28] studied the vibrational frequency of orthotropic monolayer graphene sheets embedded in an elastic medium under the effect of the change of temperature, or the solution for the vibration of orthotropic rectangular nanoplates under thermal effect and the elastic medium is obtained with using GDQM. Liu et al [29] studied buckling and post-buckling behaviors of piezoelectric nanoplates subjected to combined thermo-electro-mechanical charges based on non-local theory, Mindlin's plate theory and von Karman's geometric nonlinearity. Arefi and Zenkour [30] presented the thermo-electro-magneto-elastic bending analysis of a three-layer sandwich nanoplate based on 
Pasternak's base based on Kirchhoff plate theory and non-local theory. Askari et al [31] presented a mathematical model for the vibrations of nanoplates postulating small scale effect and nonlinear elastic foundation, the mathematical model for the vibrations of nanoplates embraces a term relevant to the added mass. The principal mode of nanoplate's oscillation is elicited using Galerkin's method. Barati et al [32] has modeled a double-layer nanoplate with a weighted composition according to the non-local deformation gradient theory (NSGT). The proposed nanoplate modeling incorporates a non-local stress field parameter as well as a length scale parameter related to the strain gradient. Bochkarev [33] showed how, using the introduced efficient modules, the classical form of state equations and the potential energy of a deformed nanoplate. Ebrahimi and Barati [34] studied the thermo-electro-mechanical vibration behavior of size-dependent flexoelectric nanoplates on the basis of non-local elasticity and surface theories. Flexoelectricity represents the coupling between the stress gradients and the electric polarization. The flexoelectric nanoplate is exposed to uniform and linear temperature increases across the thickness and applied Eringen's non-local elasticity theory for the first time in the analysis of flexoelectric nanoplates. Farrokhabadi and Tavakolian [35] developed a mathematical model, which accounts for non-linearities due to electrostatic and Casimir attractions as well as thermal force, the model examines the dynamic response of nanoactuators based on fully blocked nanoplates and simply supported. Karličić et al [36] used non-local theory to study the free vibration behavior of VOMNPS incorporated in a viscoelastic medium and subjected to the magnetic field in the plane and the non-local theory of the Kirchhoff plate, induced the Lorentz forces in the nanoplates by a magnetic field applied. Nematollahi et al [37] used a higher order, non-local deformation gradient theory to study the size-dependent effects on the vibrational behavior of thin rectangular nanoplates in different thermal environments. Satish et al [38] presented the thermal vibration characteristics of nanoplates with consideration of the effects of surface elasticity and nonlocal elasticity and investigated the effect of magnetic field strength on thermal vibration properties. Shahverdi and Barati [39] modeled a nano-porous nanoplate with a dimensional composition according to the theory of nonlinear deformation gradient (NSGT), the proposed modeling of nano-plates incorporates a parameter of non-local stress field as well as a parameter of the scale of related lengths at the deformation gradient. Yang et al [40] used an analytical model dependent on the size of an electrically driven circular nanoplate and to study the coupling effects of the nonlocal parameter, the modulus of elasticity of surface and the residual stress of surface on the dynamic instability. Zhang et al [41] studied the static bending deformation of one-dimensional (1D) quasicrystal piezoelectric hexagonal (1D) nanoplates under surface electro-elastic loads on the basis of non-local elasticity theory, Ansari et al [42] studied the bending of embedded nanoplates subjected to a uniform static load as part of Eringen's non-local integral model. Chen et al [43] used the non-local theory of Kirchhoff's plate to describe the motion of graphene sheets. The frequency responses of single-layer graphene sheets are investigated by the non-local FEM and investigated the structural intensity of the thermal load nanoplate structure by the non-local deformation gradient theory. Mohseni et al [44] developed a thick plate model for the analysis of free vibrations of rectangular FG micro-plates using the modified couple stress theory and used a variational formulation based on the Hamilton principle to obtain the equations of motion and boundary conditions. Karami et al [45] examined in their study for the first time that guided waves in rectangular FG nanoplates imperfectly tightened on the basis of non-local elasticity theory and first-order shear deformation theory. The non-local parameter is used to examine the size-dependent wave propagation behavior of FG nanoplates. Yang et al [46] obtained a thermoelastic solution for a 2D QC nanoplate subjected to surface temperature loading from the non-local theory. Lin et al [47] presented the clamped-clamped circular nanoplate model and a hybrid differential / finite difference method for studying the nonlinear behavior and 
pull-in instability of a NEMS device. Pang et al [48] studied the vibration-dependent properties of the size of a rectangular viscoelastic nanoplate taking into account the high order surface stress effect. Norouzzadeh and Ansari [49] presented a size-dependent analysis of surface stress and non-local influences on the free vibration characteristics of rectangular and circular nanoplates. Shahrbabaki [50] presented the potential functions for the representation of the Helmholtz displacement vector of the three-dimensional free vibratory analysis of the simply supported nanoplate and the wave propagation in a three-dimensional infinite nonlocal solid. In addition, he developed new trigonometric series as approximation functions in Galerkin's approach to analyzing three-dimensional nanoplates with various boundary conditions. Zenkour [51] has developed a non-local theory of elasticity for the thermoelastic vibration of nanoplates. Belkorissat et al. [52] studied a free vibration characteristics of FG nano-scale plates using a new nonlocal hyperbolic refined plate theory.

This work presents a study of the free vibration of functionally graded nanoplates simply supported using a four-variable shear strain plate theory. Current solutions of the dimensionless frequency are compared with those of the finite element method presented by Zargaripoor et al [53] and the solutions obtained by other researchers. small effects are introduced using non-local elasticity theory. In this paper the effect of the material property, aspect ratio, and other parameters is presented.

\section{Mathematical formulation}

\subsection{Theory of nonlocal elasticity}

Using the theory of non-local elasticity, presented by Eringen [54], the effects of small inter-atomic forces are considered as material parameters in the constitutive equation:

$$
\left(1-\mu^{2} \nabla^{2}\right) \sigma_{i j}^{N L}=\sigma_{i j}^{L}
$$

where: $i, j=x, y, z$

$\nabla^{2}=\frac{\partial^{2}}{\partial x^{2}}+\frac{\partial^{2}}{\partial y^{2}}$ the Laplacian operator

Superscript $(N L, L)$ : represents respectively non-local and local stress; $\mu$ : The nonlocal parameter represents the small-scale effect $\left(\mu=e_{0} a\right)$

The non-local constitutive equations of a non-local FGM plate can be written as:

$$
\left(1-\left(e_{0} a\right)^{2}\left(\frac{\partial^{2}}{\partial x^{2}}+\frac{\partial^{2}}{\partial y^{2}}\right)\right)\left\{\begin{array}{l}
\sigma_{x} \\
\sigma_{y} \\
\tau_{x y} \\
\tau_{y z} \\
\tau_{x z}
\end{array}\right\}=\left[\begin{array}{ccccc}
Q_{11} & Q_{12} & 0 & 0 & 0 \\
Q_{12} & Q_{22} & 0 & 0 & 0 \\
0 & 0 & Q_{66} & 0 & 0 \\
0 & 0 & 0 & Q_{44} & 0 \\
0 & 0 & 0 & 0 & Q_{55}
\end{array}\right]\left\{\begin{array}{l}
\sigma_{x} \\
\sigma_{y} \\
\tau_{x y} \\
\tau_{y z} \\
\tau_{x z}
\end{array}\right\}
$$

where: $\quad a:$ is an internal characteristic length.

$e_{0}:$ is a constant.

$\left(\sigma_{x}, \sigma_{y}, \tau_{x y}, \tau_{y z}, \tau_{y x}\right)$ and $\left(\varepsilon_{x}, \varepsilon_{y}, \gamma_{x y}, \gamma_{y z}, \gamma_{y x}\right)$ are the stress and strain components, respectively.

The coefficients $Q_{i j}$ of the stiffness matrix are defined as follows:

$$
Q_{11}=Q_{22}=\frac{E(z)}{1-v^{2}}, Q_{12}=Q_{21}=\frac{v E(z)}{1-v^{2}}, Q_{44}=Q_{55}=Q_{66}=\frac{E(z)}{2(1+v)}
$$

or: E: Young's modulus, v: Poisson's ratio 


\subsection{Geometrical configuration}

Consider a rectangular plate having the following dimensions:

- $\quad$ The length $(a)$

- $\quad$ The width $(b)$

- The thickness $(h)$

As indicated in (Fig.1). The chosen coordinate system (x, y, z) is placed in the middle of the plate and the coordinate parameters are such that:

- $\quad 0 \leq x \leq a$

- $0 \leq y \leq b$

- $\quad-\frac{h}{2} \leq h \leq \frac{h}{2}$

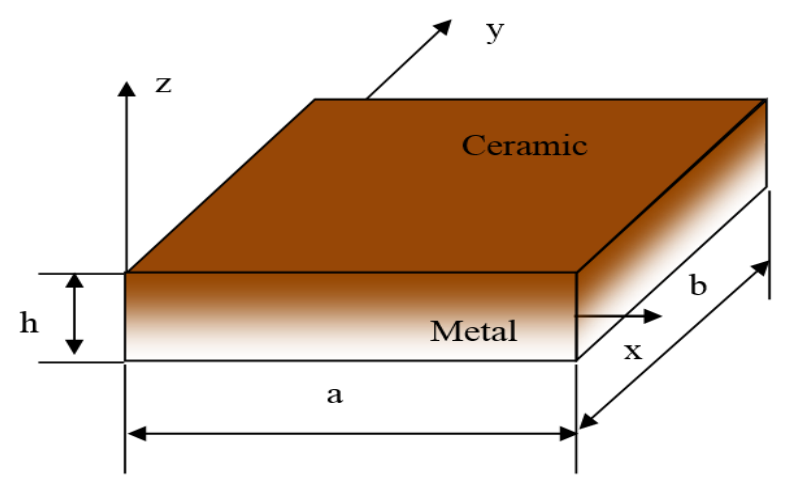

Fig. 1 Geometry of the FGM nanoplate

The material properties $\mathrm{P}$ of the P-FG plate such as the modulus of elasticity E, vary in the thickness direction $\mathrm{z}$ according to a linear rule of the mixture as:

$$
E(z)=E_{m}+\left(E_{c}-E_{m}\right)\left(\frac{z}{h}+\frac{1}{2}\right)^{P}
$$

\section{$3 \quad$ Kinematics and constituent equations}

Using the shear strain plate theory of the current model is only four variables $\left(u_{0}, v_{0}, w_{b}, w_{s}\right)$ can be given as:

$$
\begin{aligned}
& u(x, y, z)=u(x, y)-z \frac{\partial w_{b}}{\partial x}+f(z) \frac{\partial w_{s}}{\partial x} \\
& v(x, y, z)=v(x, y)-z \frac{\partial w_{b}}{\partial y}+f(z) \frac{\partial w_{s}}{\partial y} \\
& w(x, y, z)=w_{b}(x, y)+w_{s}(x, y)
\end{aligned}
$$

where $f(z)$ chosen as Sobhy and Alotebi [55] $f(z)=\left(z /\left(1+4 z^{2} / h^{2}\right)\right)-z$ using the displacement fields, the strain equation could be written as follows:

$$
\begin{aligned}
& \left\{\begin{array}{l}
\varepsilon_{x x} \\
\varepsilon_{y y} \\
\gamma_{x y}
\end{array}\right\}=\left\{\begin{array}{c}
\varepsilon_{x}^{0} \\
\varepsilon_{y}^{0} \\
\gamma_{x y}^{0}
\end{array}\right\}+z\left\{\begin{array}{c}
k_{x}^{b} \\
k_{y}^{b} \\
k_{x y}^{b}
\end{array}\right\}+f(z)\left\{\begin{array}{c}
\eta_{x}^{b} \\
\eta_{y}^{b} \\
\eta_{x y}^{b}
\end{array}\right\} \\
& \varepsilon_{z}=0, \quad\left\{\begin{array}{l}
\gamma_{x z} \\
\gamma_{y z}
\end{array}\right\}=g(z)\left\{\begin{array}{l}
\gamma_{x y}^{s} \\
\gamma_{x y}^{s}
\end{array}\right\}
\end{aligned}
$$

with 


$$
\begin{aligned}
& \varepsilon_{x}^{0}=\frac{\partial u}{\partial x}, \varepsilon_{y}^{0}=\frac{\partial v}{\partial y}, \gamma_{x y}^{0}=\frac{\partial u}{\partial y}+\frac{\partial v}{\partial x} k_{x}^{b}=-\frac{\partial^{2} w_{b}}{\partial x^{2}} \\
& k_{y}^{b}=-\frac{\partial^{2} w_{b}}{\partial y^{2}} k_{x y}^{b}=-2 \frac{\partial^{2} w_{b}}{\partial x \partial y}, k_{x}^{s}=\frac{\partial^{2} w_{s}}{\partial x^{2}} \\
& k_{y}^{s}=\frac{\partial^{2} w_{s}}{\partial y^{2}}, k_{x y}^{s}=2 \frac{\partial^{2} w_{s}}{\partial x \partial y}, \gamma_{y z}^{s}=\frac{\partial w_{s}}{\partial y}, \gamma_{x z}^{s}=\frac{\partial w_{s}}{\partial x} \\
& g=\frac{\partial f}{\partial z}+1
\end{aligned}
$$

\section{Governing equations}

The equations of equilibrium are deduced using the principle of virtual displacements, are given by:

$$
\begin{array}{r}
\int_{h / 2}^{-h / 2} \int_{S}\left[\sigma_{X} \delta \varepsilon_{X}+\sigma_{y} \delta \varepsilon_{y}+\tau_{x y} \delta \gamma_{x y}+\tau_{y z} \delta \gamma_{y z}+\tau_{x z} \delta \gamma_{x z}\right] d S d z \\
-\int_{h / 2}^{-h / 2} \int_{S} \rho\left[u " \delta u+v^{\prime \prime} \delta v+w^{\prime \prime} \delta w\right] d S d z d t=0
\end{array}
$$

By replacing Eqs. (5), (6) and (7) in Eq. (8) and integrating Eq. (8) into the plate thickness can be rewritten as follows:

$$
\begin{aligned}
\int_{s}\left[N_{x} \delta \varepsilon_{x}^{0}+\right. & N_{y} \delta \varepsilon_{y}^{0}+N_{x y} \delta \varepsilon_{x y}^{0}+M_{x} \delta k_{x}^{b}+M_{y} \delta k_{y}^{b}+M_{x y} \delta k_{x y}^{b}+S_{x} \delta k_{x}^{s} \\
& \left.+S_{y} \delta k_{y}^{s}+S_{x y} \delta k_{x y}^{s}+Q_{y z} \delta \gamma_{y z}^{s}+Q_{x z} \delta \gamma_{x z}^{s}\right] d s \\
& -\int_{-\frac{h}{2}}^{\frac{h}{2}} \int_{s} \rho\left[\left(u_{u}-z w_{x x u}^{b}+f w_{x x u}^{s}\right) \delta\left(u-z w_{x x}^{b}+f w_{x x}^{s}\right)+\right. \\
& \left(v_{u}-z x_{y y u}^{b}+f w_{y y u}^{s}\right) \delta\left(v-z x_{y y}^{b}+f w_{y y}^{s}\right) \times\left(w_{u}^{b}+w_{u}^{s}\right) \delta\left(w^{b}\right. \\
& \left.\left.+w^{s}\right)\right] d S d z .
\end{aligned}
$$

Where the resulting constraints $\mathrm{N}, \mathrm{M}$, and $\mathrm{S}$ are defined by:

$$
\begin{aligned}
& \left(N_{x}, N_{y}, N_{x y}\right)=\int_{-h / 2}^{h / 2}\left(\sigma_{x}, \sigma_{y}, \tau_{x y}\right) d z \\
& \left(M_{x}, M_{y}, M_{x y}\right)=\int_{-h / 2}^{h / 2}\left(\sigma_{x}, \sigma_{y}, \tau_{x y}\right) z d z \\
& \left(S_{x}, S_{y}, S_{x y}\right)=\int_{-h / 2}^{h / 2}\left(\sigma_{x}, \sigma_{y}, \tau_{x y}\right) f d z \\
& \left(Q_{y z}, Q_{y z}\right)=\int_{-h / 2}^{h / 2}\left(\tau_{x z}, \tau_{y z}\right) g d z
\end{aligned}
$$

Substituting Eq. (6) in Eq. (10) and integrating across the thickness of the plate, the constraints are given as follows:

$$
\left\{\begin{array}{l}
N \\
M \\
S
\end{array}\right\}-\left(e_{0} a\right)^{2}\left(\frac{\partial^{2}}{\partial x^{2}}+\frac{\partial^{2}}{\partial y^{2}}\right)\left\{\begin{array}{l}
N \\
M \\
S
\end{array}\right\}=\left[\begin{array}{ccc}
A_{i j} & B_{i j} & B_{i j}^{f} \\
B_{i j} & D_{i j} & D_{i j}^{f} \\
B_{i j}^{f} & D_{i j}^{f} & F_{i j}^{f}
\end{array}\right]\left\{\begin{array}{l}
\varepsilon \\
\kappa \\
\eta
\end{array}\right\}
$$




$$
Q-\left(e_{0} a\right)^{2}\left(\frac{\partial^{2}}{\partial x^{2}}+\frac{\partial^{2}}{\partial y^{2}}\right) Q=\gamma A_{i j}^{f}
$$

In which $N, M$ and $S$ are axial, bending and shear moments respectively. Where: $A_{i j}, B_{i j}$, etc., are the rigidity of the plate, defined by:

$$
\begin{aligned}
& \left\{A_{i j}, B_{i j}, D_{i j}\right\}=\int_{-h / 2}^{h / 2}\left\{1, z, z^{2}\right\} Q_{i j} d z, \quad(i, j=1,2,6) \\
& \left\{B_{i j}^{f}, D_{i j}^{f}, F_{i j}^{f}\right\}=\int_{-h / 2}^{h / 2}\left\{f(z), z f(z), f(z)^{2}\right\} Q_{i j} d z, \quad(i, j=1,2,6) \\
& \left\{A_{i j}^{f}\right\}=\int_{-h / 2}^{h / 2}\left\{g^{2}\right\} Q_{i j} d z, \quad(i, j=4,5)
\end{aligned}
$$

The equilibrium equations can be derived from Eq. (9) by integrating the displacement gradients by parts and separately fix the coefficients $\delta u, \delta v, \delta w_{b}$ and $\delta w_{s}$.

Thus, equilibrium equations can be obtained corresponding to the current four-variable non-local elasticity theory for an FGM plate:

$$
\begin{gathered}
\delta u: \frac{\partial N_{X}}{\partial x}+\frac{\partial N_{x y}}{\partial y}=I_{1} \frac{\partial^{2} u}{\partial t^{2}}-I_{2} \frac{\partial^{3} w_{b}}{\partial x \partial t^{2}}+I_{4} \frac{\partial^{3} w_{s}}{\partial x \partial t^{2}} \\
\delta v: \frac{\partial N_{X y}}{\partial x}+\frac{\partial N_{y}}{\partial y}=I_{1} \frac{\partial^{2} v}{\partial t^{2}}-I_{2} \frac{\partial^{3} w_{b}}{\partial y \partial t^{2}}+I_{4} \frac{\partial^{3} w_{s}}{\partial y \partial t^{2}} \\
\delta w_{b}: \frac{\partial^{2} M_{X}}{\partial x^{2}}+2 \frac{\partial^{2} M_{X y}}{\partial x \partial y}+\frac{\partial^{2} M_{y}}{\partial y^{2}} \\
=I_{2}\left(\frac{\partial^{3} u}{\partial x \partial t^{2}}+\frac{\partial^{3} v}{\partial x \partial t^{2}}\right)-I_{3}\left(\frac{\partial^{4} w_{b}}{\partial x^{2} \partial t^{2}}+\frac{\partial^{4} w_{b}}{\partial y^{2} \partial t^{2}}\right) \\
+I_{5}\left(\frac{\partial^{4} w_{s}}{\partial x^{2} \partial t^{2}}+\frac{\partial^{4} w_{s}}{\partial y^{2} \partial t^{2}}\right)+I_{1}\left(\frac{\partial^{2} w_{b}}{\partial t^{2}}+\frac{\partial^{2} w_{s}}{\partial t^{2}}\right) \\
\delta w_{s}: \frac{\partial^{2} S_{X}}{\partial x^{2}}+2 \frac{\partial^{2} S_{X y}+\frac{\partial^{2} S_{y}}{\partial x}-\frac{Q_{X z}}{\partial y}-\frac{\partial Q_{y z}}{\partial y}}{\partial y} \\
=I_{4}\left(\frac{\partial^{3} u}{\partial x \partial t^{2}}+\frac{\partial^{3} v}{\partial x \partial t^{2}}\right)-I_{5}\left(\frac{\partial^{4} w_{b}}{\partial x^{2} \partial t^{2}}+\frac{\partial^{4} w_{b}}{\partial y^{2} \partial t^{2}}\right) \\
+I_{6}\left(\frac{\partial^{4} w_{S}}{\partial x^{2} \partial t^{2}}+\frac{\partial^{4} w_{S}}{\partial y^{2} \partial t^{2}}\right)+I_{1}\left(\frac{\partial^{2} w_{b}}{\partial t^{2}}+\frac{\partial^{2} w_{s}}{\partial t^{2}}\right)
\end{gathered}
$$

Eqs. (14-17) can be expressed in terms of displacements $\left(u, v, w_{b}, w_{s}\right)$ by substituting the results of Eqs. (11) and (12). For nonlocal plates, the equilibrium equations take the form: 


$$
\begin{aligned}
& {\left[-I_{1} \frac{\partial^{2} u}{\partial t^{2} \partial x^{2}}+I_{2} \frac{\partial^{3} w_{b}}{\partial t^{2} \partial x^{3}}-I_{4} \frac{\partial^{3} w_{s}}{\partial t^{2} \partial x^{3}}-I_{1} \frac{\partial^{2} u}{\partial t^{2} \partial y^{2}}+I_{2} \frac{\partial^{3} w_{b}}{\partial x \partial y^{2} \partial t^{2}}\right.} \\
& \left.-I_{4} \frac{\partial^{3} w_{s}}{\partial x \partial y^{2} \partial t^{2}}\right]\left(e_{0} a\right)^{2}+\left(I_{1} \frac{\partial^{2} u}{\partial t^{2}}-I_{2} \frac{\partial^{3} w_{b}}{\partial x \partial t^{2}}+I_{4} \frac{\partial^{3} w_{s}}{\partial x \partial t^{2}}\right) \\
& =A_{11} \frac{\partial^{2} u}{\partial x^{2}}+A_{66} \frac{\partial^{2} u}{\partial y^{2}}+\left(A_{12}+A_{66}\right) \frac{\partial^{2} v}{\partial x \partial y}-B_{11} \frac{\partial^{3} w_{b}}{\partial x^{3}} \\
& =-\left(B_{12}+2 B_{66}\right) \frac{\partial^{3} w_{b}}{\partial y^{2} \partial x}+B_{11}^{f} \frac{\partial^{3} w_{s}}{\partial x^{3}}+\left(B_{12}^{f}+2 B_{66}^{f}\right) \frac{\partial^{3} w_{s}}{\partial y^{2} \partial x} \\
& {\left[-I_{1} \frac{\partial^{2} u}{\partial t^{2} \partial x^{2}}+I_{2} \frac{\partial^{3} w_{b}}{\partial t^{2} \partial x^{3}}-I_{4} \frac{\partial^{3} w_{s}}{\partial t^{2} \partial x^{3}}-I_{1} \frac{\partial^{2} u}{\partial t^{2} \partial y^{2}}+I_{2} \frac{\partial^{3} w_{b}}{\partial x \partial y^{2} \partial t^{2}}\right.} \\
& \left.-I_{4} \frac{\partial^{3} w_{s}}{\partial x \partial y^{2} \partial t^{2}}\right]\left(e_{0} a\right)^{2}+\left(I_{1} \frac{\partial^{2} u}{\partial t^{2}}-I_{2} \frac{\partial^{3} w_{b}}{\partial x \partial t^{2}}+I_{4} \frac{\partial^{3} w_{s}}{\partial x \partial t^{2}}\right) \\
& =A_{11} \frac{\partial^{2} u}{\partial x^{2}}+A_{66} \frac{\partial^{2} u}{\partial y^{2}}+\left(A_{12}+A_{66}\right) \frac{\partial^{2} v}{\partial x \partial y}-B_{11} \frac{\partial^{3} w_{b}}{\partial x^{3}}-\left(B_{12}\right. \\
& \left.+2 B_{66}\right) \frac{\partial^{3} w_{b}}{\partial y^{2} \partial x}+B_{11}^{f} \frac{\partial^{3} w_{s}}{\partial x^{3}}+\left(B_{12}^{f}+2 B_{66}^{f}\right) \frac{\partial^{3} w_{s}}{\partial y^{2} \partial x}
\end{aligned}
$$

\section{$5 \quad$ Analytical solutions}

Rectangular plates are generally classified according to the type of substrate used. We consider here the exact solution for an FGM plate simply supported. Following the Navier solution procedure, we assume that the form of the following solution for $\left(u, v, w_{b}, w_{s}\right)$ satisfies the boundary conditions:

$$
\left\{\begin{array}{c}
u \\
v \\
w_{b} \\
w_{s}
\end{array}\right\}=\left\{\begin{array}{c}
U \cos (\alpha x) \sin (\beta y) \\
V \sin (\alpha x) \cos (\beta y) \\
W_{b} \sin (\alpha x) \sin (\beta y) \\
W_{s} \sin (\alpha x) \sin (\beta y)
\end{array}\right\} e^{i \omega t}
$$

where: $\left(u, v, w_{b}, w_{s}\right)$ are arbitrary parameters to be determined under the condition that the solution satisfies the equilibrium equations noting that $\left(\alpha=\frac{m \pi}{a}, \beta=\frac{n \pi}{b}\right)$.

The below eigenvalue equations for any fixed value of $m$ and $n$, for free vibration problem:

$$
\left([k]-\omega^{2}[k]\right)\{\Delta\}=\{0\},
$$

or $\{\Delta\}$ : designates the columns, and $[k]$ is the stiffness matrix and $[M]$ refers to the mass matrix in the case of free vibration:

$$
\begin{aligned}
& \{\Delta\}=\left\{\begin{array}{l}
u_{m n} \\
v_{m n} \\
w_{b m n} \\
w_{s m n}
\end{array}\right\},[k]=\left[\begin{array}{llll}
K_{11} & K_{12} & K_{13} & K_{14} \\
K_{12} & K_{22} & K_{23} & K_{24} \\
K_{13} & K_{23} & K_{33} & K_{34} \\
K_{14} & K_{24} & K_{34} & K_{44}
\end{array}\right], \\
& {[M]=\left[\begin{array}{llll}
M_{11} & M_{12} & M_{13} & M_{14} \\
M_{12} & M_{22} & M_{23} & M_{24} \\
M_{13} & M_{23} & M_{33} & M_{34} \\
M_{14} & M_{24} & M_{34} & M_{44}
\end{array}\right]}
\end{aligned}
$$

The elements $K_{i j}=K_{j i} ; M_{i j}=M_{j i}$ of the coefficient matrix $[k]$ and $[M]$ 


$$
\begin{aligned}
& K_{11}=\alpha^{2} A_{11}+\beta^{2} A_{66} \\
& K_{12}=\alpha \beta\left(A_{12}+A_{66}\right) \\
& K_{13}=-B_{11} \alpha^{3}-\alpha \beta^{2}\left(B_{12}+2 B_{66}\right) \\
& K_{14}=B_{11}^{f} \alpha^{3}+\alpha \beta^{2}\left(B_{12}^{f}+2 B_{66}^{f}\right) \\
& K_{22}=A_{22} \beta^{2}+A_{66} \alpha^{2} \\
& K_{23}=-B_{22} \beta^{3}-\alpha^{2} \beta\left(B_{12}+2 B_{66}\right) \\
& K_{24}=B_{22}^{f} \beta^{3}+\alpha^{2} \beta\left(B_{12}^{f}+2 B_{66}^{f}\right) \\
& K_{33}=D_{11} \alpha^{4}+D_{22} \beta^{4}+2\left(D_{12}+2 D_{66}\right) \alpha^{2} \beta^{2} \\
& K_{34}=-D_{22}^{f} \beta^{4}-D_{11}^{f} \alpha^{4}-2\left(D_{12}^{f}+2 D_{66}^{f}\right) \alpha^{2} \beta^{2} \\
& K_{44}=-f_{11}^{f} \alpha^{4}-f_{22}^{f} \beta^{4}-2\left(f_{12}^{f}+2 f_{66}^{f}\right) \alpha^{2} \beta^{2}-A_{44}^{f} \beta^{2}-A_{55}^{f} \alpha^{2} \\
m_{11}= & \left(1+\beta\left(\alpha^{2} \beta^{2}\right)\right) I_{1} \\
m_{12}= & 0 \\
m_{13}= & \left(-\beta \alpha^{3}-\left(\beta^{3}+1\right) \alpha\right) I_{2} \\
m_{14}= & \left(\beta \alpha^{3}+\left(\beta^{3}+1\right) \alpha\right) I_{4} \\
m_{21}= & 0 \\
m_{22}= & \left(1+\beta\left(\alpha^{2}+\beta^{2}\right)\right) I_{1} \\
m_{23}= & -\left(\beta^{2} \alpha^{2}+\beta^{4}-\beta\right) I_{2} \\
m_{24}= & \left(\beta^{2} \alpha^{2}+\beta^{4}-\beta\right) I_{4} \\
m_{31}= & I_{2}\left(\left(e_{0} a\right)^{2}-1\right) \\
m_{33}= & \left(\alpha^{2} \beta+\beta^{3}+1\right) I_{1}+\left(\alpha^{2}+\beta^{2}+\beta\left(\alpha^{4}+\beta^{2} \alpha^{2}\right)+\beta\left(\beta^{2} \alpha^{2}+\beta^{4}\right)\right) I_{3} \\
m_{34}= & \left(\alpha^{2} \beta+\beta^{3}+1\right) I_{1}-\left(\alpha^{2}+\beta^{2}+\beta\left(\alpha^{4}+\beta^{2} \alpha^{2}\right)+\beta\left(\beta^{2} \alpha^{2}+\beta^{4}\right)\right) I_{5} \\
m_{44}= & \left(1+\beta\left(\alpha^{2}+\beta^{2}\right)\right) I_{1}+\left(\alpha^{2}+\beta^{2}+\beta\left(\alpha^{4}+2 \beta^{2} \alpha^{2}+\beta^{4}\right)\right) I_{6} \\
&
\end{aligned}
$$

\section{Numerical results and discussion}

To verify the accuracy of the current theory of FGM nanoparticles, comparisons are made between the results obtained from the current theory and those obtained by the finite element solution of Zargaripoor et al [53] as shown in the tables below.

The material properties used in the present study are as follows:

as metal (SUS 304):

$$
E_{m}=201.04 \mathrm{GPa}, \quad \rho_{m}=8166 \mathrm{~kg} / \mathrm{m}^{3}, v_{m}=0.3
$$

as ceramic $(\mathrm{Si} 3 \mathrm{~N} 4)$ :

$$
E_{c}=348.46 \mathrm{GPa}, \quad \rho_{c}=2370 \mathrm{~kg} / \mathrm{m}^{3}, \quad v_{m}=0.3
$$

Where E, $v, \rho$ is Young's modulus, the Poisson's ratio and the density of the plate. The following dimensionless parameters are used:

$$
\eta=\frac{a}{b}, \delta=\frac{h}{a}, \bar{\omega}=\omega h \sqrt{\frac{\rho_{c}}{G_{c}}}, F r=\frac{\bar{\omega}_{N L}}{\omega_{L}}
$$

In which $\omega_{L}$ is the dimensionless frequency corresponding to the non-local parameter $\mu=0$. 
Table 1. Comparison of dimensionless frequency $\bar{\omega}=\omega h \sqrt{\frac{\rho_{c}}{G_{c}}}$ for FG square nanoplate

\begin{tabular}{llll}
\multicolumn{4}{c}{$(\eta=1, \delta=0.1, n=5)$} \\
\hline \multirow{2}{*}{ Method } & $\mu=0$ & $\mu=1$ & $\mu=2$ \\
\cline { 2 - 4 } Zargaripoor et al [53] & 0.0444 & 0.0405 & 0.0376 \\
Aghababaei and Reddy [56] & 0.0441 & 0.0403 & 0.0374 \\
Present & $\mathbf{0 . 0 4 4 2}$ & $\mathbf{0 . 0 4 0 4}$ & $\mathbf{0 . 0 3 7 4}$ \\
\hline
\end{tabular}

Table 1 represents a comparison of the dimensionless frequencies obtained by the present theory, the finite element solution of Zargaripoor et al [53] and the results obtained by Aghababaei and Reddy [56] for the simply supported FG nanoplates. it can be seen that the results obtained with the present theory and the finite element method have a considerable precision because they are very close and almost the same. Table 2 gives a comparison of the dimensionless frequencies obtained by the present theory and that of the finite element method presented by Zargaripoor et al [53] and other results found in the literature for simply supported FG nanoplates. this table shows a good agreement between the results.

Table 2. Comparison of dimensionless frequency $\bar{\omega}=\omega h \sqrt{\frac{\rho}{G}}$ for a simply supported nanoplate.

\begin{tabular}{ccccc}
\hline \hline \multirow{2}{*}{$\eta$} & \multirow{2}{*}{$\delta$} & $\mu$ & \multicolumn{3}{c}{ Dimensionless Frequency } \\
\cline { 3 - 5 } & & Present & Zargaripoor et al [53] & Aghababaei and Reddy [56] \\
\hline & 0 & $\mathbf{0 . 0 9 3 1}$ & 0.0930 & 0.0935 \\
10 & 1 & $\mathbf{0 . 0 8 5 1}$ & 0.0850 & 0.0854 \\
& 2 & $\mathbf{0 . 0 7 8 9}$ & 0.0788 & 0.0791 \\
\hline & 0 & $\mathbf{0 . 0 2 3 9}$ & 0.0239 & 0.0239 \\
20 & 1 & $\mathbf{0 . 0 2 1 8}$ & 0.0218 & 0.0218 \\
& 2 & $\mathbf{0 . 0 2 0 2}$ & 0.0202 & 0.0202 \\
\hline & 0 & $\mathbf{0 . 0 5 8 9}$ & 0.0589 & 0.0591 \\
10 & 1 & $\mathbf{0 . 0 5 5 6}$ & 0.0556 & 0.0557 \\
& 2 & $\mathbf{0 . 0 5 2 8}$ & 0.0527 & 0.0529 \\
\hline & 0 & $\mathbf{0 . 0 1 5 0}$ & 0.0150 & 0.0150 \\
20 & 1 & $\mathbf{0 . 0 1 4 1}$ & 0.0141 & 0.0141 \\
& 2 & $\mathbf{0 . 0 1 3 4}$ & 0.0134 & 0.0134 \\
\hline
\end{tabular}

Table 3 gives a comparison of the results of the dimensionless frequency found by the present theory and that of the finite element method presented by Zargaripoor et al [53] for different modes of vibration of simply supported nanoplate.

Figure 2 shows the dimensionless frequency variation of a simply supported FG nanoplate, based on the variation of non-local parameters for different power law index values.

In this figure, it can be seen that when the non-local parameters and the power law index increase, the non-dimensional frequency decreases. 
It can be seen that for the lower power law index, the dimensionless frequency value is higher, because by increasing the power law index, the plate property reaches the metal, and therefore its rigidity decreases. Thus, for a higher power law index, the value of the dimensionless frequency is lower.

The effect of the aspect ratio on the frequency ratio of simply supported FG nanoplate is illustrated in Figure 3. It can be seen that if the aspect ratio of the plate increases, the frequency ratio decreases.

Figure 4 shows the effects of the aspect ratio on non-dimensional frequency for different values of non-local parameters. It can be seen that by increasing the aspect ratio, the frequency increases for all non-local parameters. It can be seen that for higher aspect ratios, the influence of non-local parameters increases.

Table 3. Comparison of dimensionless frequency $\bar{\omega}=\omega h \sqrt{\frac{\rho_{c}}{G_{c}}}$ for FG simply supported square nanoplate $(\eta=1, \delta=0.1)$

\begin{tabular}{|c|c|c|c|c|c|c|c|c|c|}
\hline \multirow{3}{*}{$\begin{array}{c}\text { Power } \\
\text { law } \\
\text { index }\end{array}$} & \multirow{3}{*}{$\begin{array}{l}\text { Nonlocal } \\
\text { parameter }\end{array}$} & \multicolumn{8}{|c|}{ Dimensionless Frequency } \\
\hline & & \multicolumn{2}{|c|}{ Mode1 $(1,1)$} & \multicolumn{2}{|c|}{ Mode2 $(1,2)$} & \multicolumn{2}{|c|}{ Mode3 $(2,1)$} & \multicolumn{2}{|c|}{ Mode4 $(2,2)$} \\
\hline & & Present & $\begin{array}{c}\text { Zargaripoor } \\
\text { et al [53] }\end{array}$ & Present & $\begin{array}{c}\text { Zargaripoor } \\
\text { et al [53] }\end{array}$ & Present & $\begin{array}{c}\text { Zargaripoor } \\
\text { et al [53] }\end{array}$ & Present & $\begin{array}{c}\text { Zargaripoor } \\
\text { et al [53] }\end{array}$ \\
\hline \multirow{3}{*}{$n=0$} & $\mu=0$ & 0.0931 & 0.0930 & 0.2226 & 0.2225 & 0.2226 & 0.2225 & 0.3421 & 0.3407 \\
\hline & $\mu=1$ & 0.0851 & 0.0850 & 0.1822 & 0.1820 & 0.1822 & 0.1820 & 0.2558 & 0.2547 \\
\hline & $\mu=2$ & 0.0789 & 0.0788 & 0.1579 & 0.1578 & 0.1579 & 0.1578 & 0.2130 & 0.2122 \\
\hline \multirow{3}{*}{$n=1$} & $\mu=0$ & 0.0548 & 0.0552 & 0.1309 & 0.1310 & 0.1309 & 0.1310 & 0.2011 & 0.2008 \\
\hline & $\mu=1$ & 0.0501 & 0.0504 & 0.1071 & 0.1072 & 0.1071 & 0.1072 & 0.1504 & 0.1501 \\
\hline & $\mu=2$ & 0.0464 & 0.0467 & 0.0929 & 0.0930 & 0.0929 & 0.0930 & 0.1253 & 0.1250 \\
\hline \multirow{3}{*}{$n=5$} & $\mu=0$ & 0.0442 & 0.0444 & 0.1052 & 0.1052 & 0.1052 & 0.1052 & 0.1613 & 0.1608 \\
\hline & $\mu=1$ & 0.0404 & 0.0405 & 0.0861 & 0.0861 & 0.0861 & 0.0861 & 0.1206 & 0.1202 \\
\hline & $\mu=2$ & 0.0374 & 0.0376 & 0.0746 & 0.0747 & 0.0746 & 0.0747 & 0.1005 & 0.1002 \\
\hline
\end{tabular}

The effect of the non-local parameter on the frequency ratio of the different vibration modes is shown in Figure 5.

It can be seen that for higher mode values, the effect of the non-local parameter becomes more noticeable.

The dimensionless frequency variation of the nanoplate simply press with the power law index for different non-local parameters is shown in Figure 8.

This figure shows that the increase in the power law index decreases the dimensionless frequency for all non-local parameters.

In addition, by increasing the power law index, the dimensionless frequency converges to the frequency of the metal plate. 


\section{Conclusion}

This paper shows the analysis of the vibratory behavior of simply supported FG nanoplate using a theory of four-variable shear deformation plates. This study allows seeing the effects of different parameters such as the thickness ratio, the exponent of the power law, the small effects, the aspect ratio and the mode of vibration on the behavior of nanoplates using the theory of nonlocal elasticity. The results obtained from dimensionless frequencies by the analytical solution of the present theory are compared with the results of the solution of the finite element method presented by Zargaripoor et al [53] and the other results of the literature for simply supported functionally graded nanoplates. A good agreement is found.

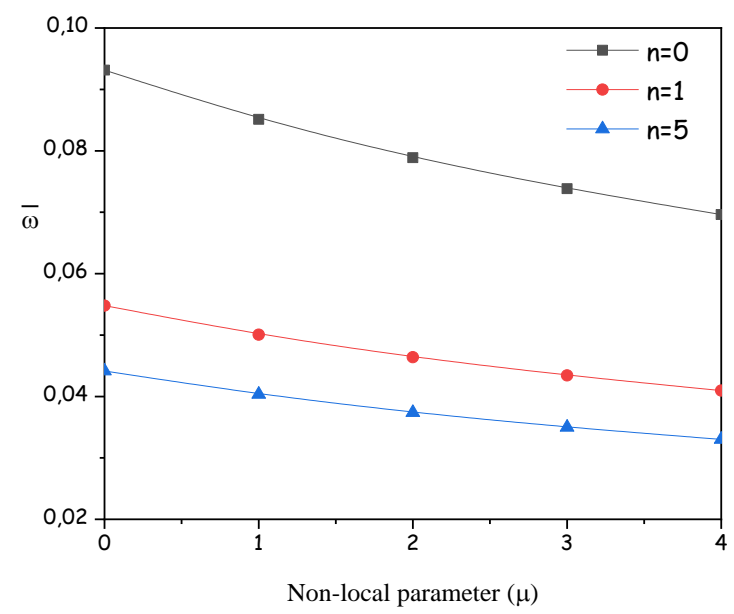

Fig. 2 Effect of nonlocal parameter on dimensionless frequency for an FG simply supported nanoplate for different power law index $(\eta=1, \delta=0.1)$

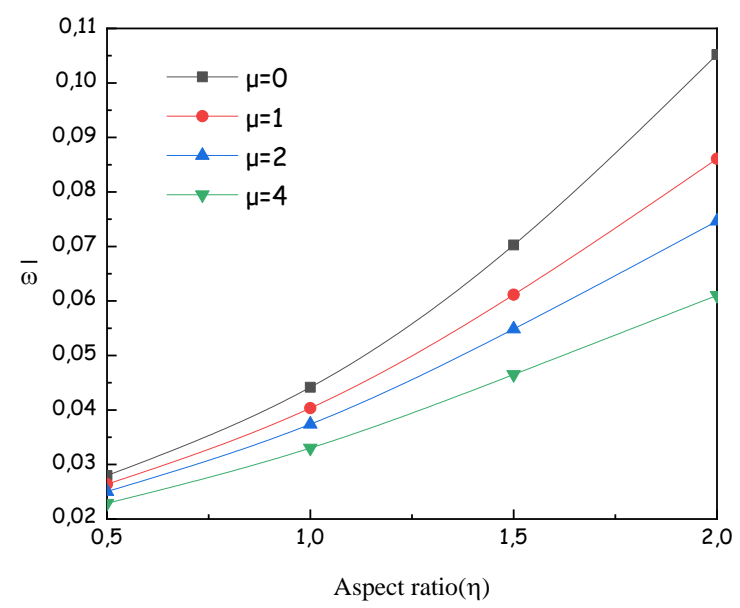

Fig. 4 Effect of aspect ratio on dimensionless

frequency for an FG simply supported nanoplate for different non-local parameters

$$
(n=5, \delta=0.1)
$$

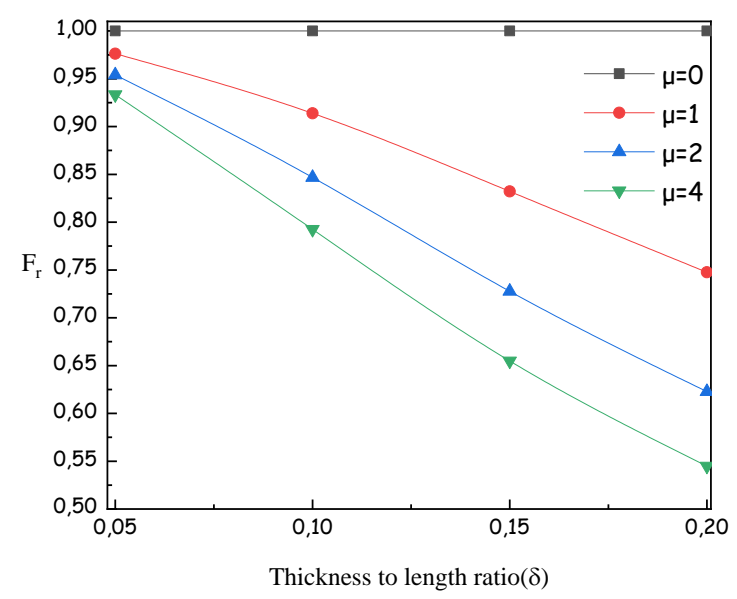

Fig. 3 Effect of thickness to length ratio on frequency ratio for an FG simply supported nanoplate for different nonlocal $\operatorname{parameters}(\eta=1, n=5)$

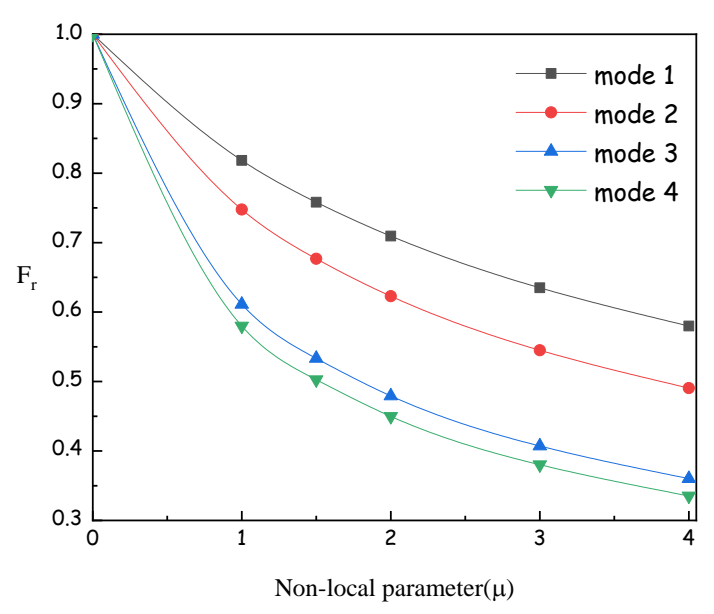

Fig. 5 Effect of non-local parameter on frequency ratio for an FG simply supported nanoplate for different mode numbers

$$
(n=5, \delta=0.1, \eta=2)
$$




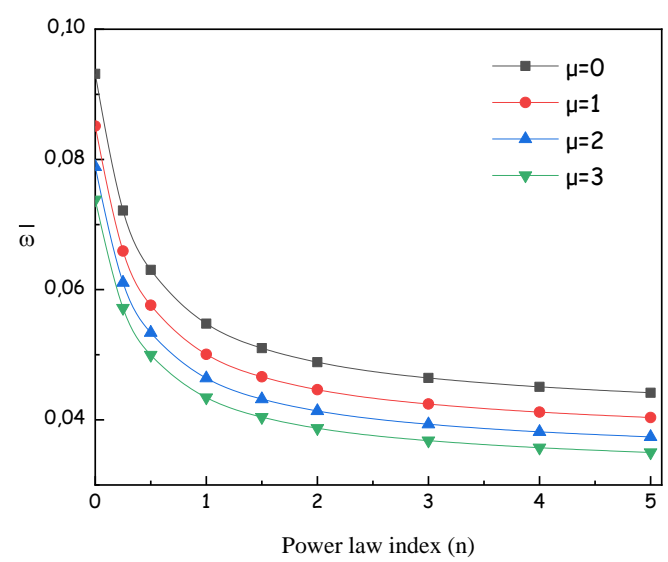

Fig. 6 Effect of the power law index on the dimensionless frequency for a simply supported square nanoplate $(\delta=0.1, \mu=1)$

It can be deduced from this study that:

- If the non-local parameter increases, the dimensionless frequency decreases.

- If the length-to-length ratio of the simply supported fg nanoplate increases, the frequency ratio decreases for different values of the non-null non-local parameter.

$\circ$ If the nonlocal parameter is zero, the increase in the thickness-to-length ratio has no influence on the dimensionless frequency.

$\circ$ If the aspect ratio of the simply supported FG nanoplate increases, the nondimensional frequency increases.

- The frequency ratio decreases with the increase of the non-local parameter for a different mode of vibration.

$\circ$ The dimensionless frequency decreases with increasing power law exponent.

\section{References}

[1] Abdelbaki, C., Ahmed, B., Houari, H., Mohammed Sid Ahmed, H., Abdelouahed, T., E. A., A. B. "Thermo-mechanical postbuckling of symmetric S-FGM plates resting on Pasternak elastic foundations using hyperbolic shear deformation theory", Structural Engineering and Mechanics 57 (4), pp. 617 - 639, 2016. DOI: 10.12989/sem.2016.57.4.617

[2] Choudhury, A., Mondal, S. C., Sarkar, S. "Effect of lamination angle and thickness on analysis of composite plate under thermo mechanical loading", Strojnícky časopis Journal of Mechanical Engineering 67 (1), pp. 5 - 22, 2017. DOI: 10.1515/scjme-20170001

[3] Abdelbaki, C., Abdelouahed, T., Habib, H., S. R, M. "Thermal buckling analysis of cross-ply laminated plates using a simplified HSDT", Smart Structures and Systems 19 (3), pp. 289 - 297, 2017. DOI: 10.12989/sss.2017.19.3.289

[4] Ebrahimi, F., Barati, M. R. "On static stability of electro-magnetically affected smart magneto-electro-elastic nanoplates", Advances in Nano Research 7 (1), pp. 63 - 75, 2019. DOI: $10.12989 /$ anr.2019.7.1.063

[5] Ebrahimi, F., Heidari, E. "Surface effects on nonlinear vibration and buckling analysis of embedded FG nanoplates via refined HOSDPT in hygrothermal environment considering physical neutral surface position", Advances in Aircraft and Spacecraft Science 5 (6), pp. 691 - 729, 2018. DOI: https: 10.12989/aas.2018.5.6.691 
[6] Elmerabet, A. H., Heireche, H., Tounsi, A., Semmah, A. "Buckling temperature of a single-walled boron nitride nanotubes using a novel nonlocal beam model", Advances in nano research 5 (1), pp. 1 - 12, 2017. DOI: 10.12989/anr.2017.5.1.001

[7] Elmossouess, B., Kebdani, S., Bouiadjra, M. B., Tounsi, A. "A novel and simple HSDT for thermal buckling response of functionally graded sandwich plates", Structural Engineering and Mechanics 62 (4), pp. $401-415$, 2017. DOI: 10.12989/sem.2017.62.4.401

[8] Houari, M. S. A., Tounsi, A., Bessaim, A., Mahmoud, S. R. "A new simple threeunknown sinusoidal shear deformation theory for functionally graded plates", Steel and Composite Structures 22 (2), pp. 257 - 276, 2016. DOI: 10.12989/scs.2016.22.2.257

[9] Karami, B., Karami, S. "Buckling analysis of nanoplate - type temperature - dependent heterogeneous materials", Advances in Nano Research 7 (1), pp. 51 - 61, 2019. DOI: 10.12989/anr.2019.7.1.051

[10] Mahjoobi, M., Bidgoli, M. R. "Vibration analysis of concrete foundation armed by silica nanoparticles based on numerical methods", Structural Engineering and Mechanics 69 (5), pp. 547 - 555, 2019. DOI: 10.12989/sem.2019.69.5.547

[11] Mohamed, B., Abed, B., Abdelmoumen Anis, B., Amel, S., Fouad, B., Abdelouahed, T., and S.R, M. "Buckling behavior of rectangular plates under uniaxial and biaxial compression", Structural Engineering and Mechanics, 70 (1), pp. 113 - 123, 2019. DOI: 10.12989/sem.2019.70.1.113

[12] Mokhtar, N., Hassen, A. A., Riadh, B., Abdelouahed, T., E.A, A. B. "Vibration response and wave propagation in FG plates resting on elastic foundations using HSDT”, Structural Engineering and Mechanics 69 (5), pp. 511 - 525, 2019. DOI: 10.12989/sem.2019.69.5.511

[13] Mokhtar, Y., Heireche, H., Bousahla, A. A., Houari, M. S. A., Tounsi, A., Mahmoud, S. R. "A novel shear deformation theory for buckling analysis of single layer graphene sheet based on nonlocal elasticity theory", Smart Structures Systems 21 (4), pp. 397 405, 2018. DOI: 10.12989/sss.2018.21.4.397

[14] Sadoun, M., Houari, M. S. A., Bakora, A., Tounsi, A., Mahmoud, S. R., Alwabli, A. S. "Vibration analysis of thick orthotropic plates using quasi 3D sinusoidal shear deformation theory", Geomechanics and Engineering 16 (2), pp.141 - 150, 2018. DOI: 10.12989/gae.2018.16.2.141

[15] Salari, E., Ashoori, A., and Vanini, S. A. S. "Porosity-dependent asymmetric thermal buckling of inhomogeneous annular nanoplates resting on elastic substrate", Advances in Nano Research 7 (1), pp. 25 - 38, 2019. DOI: 10.12989/anr.2019.7.1.025

[16] Shafiei, H., Setoodeh, A. R. "Nonlinear free vibration and post-buckling of FG-CNTRC beams on nonlinear foundation", Steel and Composite Structures 24 (1), pp. 65 - 77, 2017. DOI: $10.12989 / \mathrm{scs} .2017 .24 .1 .065$

[17] Shokravi, M. "Buckling of sandwich plates with FG-CNT-reinforced layers resting on orthotropic elastic medium using Reddy plate theory", Steel and Composite Structures 23 (6), pp. 623 - 631, 2017. DOI: 10.12989/scs.2017.23.6.623

[18] Tlidji, Y., Zidour, M., Draiche, K., Safa, A., Bourada, M., Tounsi, A., Bousahla, A. A., Mahmoud, S. R. "Vibration analysis of different material distributions of functionally graded microbeam", Structural Engineering and Mechanics 69 (6), pp. 637 - 649, 2019. DOI: 10.12989/sem.2019.69.6.637 
[19] Tounsi, A., Houari, M. S. A., Bessaim, A. "A new 3-unknowns non-polynomial plate theory for buckling and vibration of functionally graded sandwich plate", Structural Engineering and Mechanics 60 (4), pp. $547 \quad-\quad 565$, 2016. DOI: 10.12989/sem.2016.60.4.547

[20] Tu, T. M., Quoc, T. H., Long, N. V. "Bending analysis of functionally graded plates using new eight-unknown higher order shear deformation theory", Structural Engineering and Mechanics 62 (3), pp. 311 - 324, 2017. DOI: 10.12989/sem.2017.62.3.311

[21] Bocko, J., Lengvarský, P., Šarloši, J. "Buckling analysis of hetero-junction carbon nanotubes", Strojnícky časopis - Journal of Mechanical Engineering 68 (2), pp. 9 - 16, 2018. DOI: $10.2478 /$ scjme-2018-0013

[22] Bocko, J., Lengvarský, P., Pástor, M. "Estimation of Material Properties of Carbon Nanotubes Using Finite Element Method", Strojnícky časopis - Journal of Mechanical Engineering 69 (2), pp. 7 - 14, 2019. DOI: 10.2478/scjme-2019-0014

[23] Kugler, S., Fotiu, P., Murín, J. "On the Access to Transverse Shear Stiffnesses and to Stiffness Quantities for Non-Uniform Warping Torsion in FGM Beam Structures”, Strojnícky časopis - Journal of Mechanical Engineering 69 (2), pp. 27 - 56, 2019. DOI: $10.2478 /$ scjme-2019-0016

[24] Murín, J., Aminbaghai, M., Hrabovský, J. "Elastostatic analysis of the spatial FGM structures", Strojnícky časopis - Journal of Mechanical Engineering 65(1), pp. 27 - 56, 2015. DOI: $10.1515 /$ scjme-2016-0003

[25] Sapountzakis, E., Argyridi, A. "Influence of in-Plane Deformation in Higher Order Beam Theories", Strojnícky casopis-Journal of Mechanical Engineering 68 (3), pp. 77 94, 2018. DOI: $10.2478 /$ scjme-2018-0028

[26] Ansari, R., Norouzzadeh, A. "Nonlocal and surface effects on the buckling behavior of functionally graded nanoplates: An isogeometric analysis", Physica E: LowDimensional Systems and Nanostructures 84, pp. $84-97,2016$. DOI: 10.1016/j.physe.2016.05.036

[27] Banh-Thien, T., Dang-Trung, H., Le-Anh, L., Ho-Huu, V., Nguyen-Thoi, T. "Buckling analysis of non-uniform thickness nanoplates in an elastic medium using the isogeometric analysis", Composite Structures 162, pp. 182 - 193, 2017. DOI: 10.1016/j.compstruct.2016.11.092

[28] Ghadiri, M., Shafiei, N., Alavi, H. "Thermo-mechanical vibration of orthotropic cantilever and propped cantilever nanoplate using generalized differential quadrature method", Mechanics of Advanced Materials and Structures 24 (8), pp. 636 - 646, 2017. DOI: $10.1080 / 15376494.2016 .1196770$

[29] Liu, C., Ke, L.-L., Yang, J., Kitipornchai, S., Wang, Y.-S. "Buckling and post-buckling analyses of size-dependent piezoelectric nanoplates", Theoretical and Applied Mechanics Letters 6 (6), pp. 253 - 267, 2016. DOI: 10.1016/j.taml.2016.10.003

[30] Arefi, M., Zenkour, A. M. "Thermo-electro-magneto-mechanical bending behavior of size-dependent sandwich piezomagnetic nanoplates", Mechanics Research Communications 84, pp. 27 - 42, 2017. DOI: 10.1016/j.mechrescom.2017.06.002

[31] Askari, H., Jamshidifar, H., Fidan, B. "High resolution mass identification using nonlinear vibrations of nanoplates", Measurement 101, pp. 166 - 174, 2017. DOI: 10.1016/j.measurement.2017.01.012 
[32] Barati, M. R., Shahverdi, H. "Hygro-thermal vibration analysis of graded doublerefined-nanoplate systems using hybrid nonlocal stress-strain gradient theory", Composite Structures 176, pp. 982 - 995, 2017. DOI: 10.1016/j.compstruct. 2017.06.004

[33] Bochkarev, A. "Influence of boundary conditions on stiffness properties of a rectangular nanoplate", Procedia Structural Integrity 6, pp. $174-181,2017$. DOI: 10.1016/j.prostr.2017.11.027

[34] Ebrahimi, F., Barati, M. R. "Vibration analysis of size-dependent flexoelectric nanoplates incorporating surface and thermal effects." Mechanics of Advanced Materials and Structures 25 (7), pp. 611 - 621, 2018. DOI: $10.1080 / 15376494.2017 .1285464$

[35] Farrokhabadi, A., Tavakolian, F. "Size-dependent dynamic analysis of rectangular nanoplates in the presence of electrostatic, Casimir and thermal forces", Applied Mathematical Modelling 50, pp. 604 - 620, 2017. DOI: 10.1016/j.apm.2017.06.017

[36] Karličić, D., Cajić, M., Adhikari, S., Kozić, P., Murmu, T. "Vibrating nonlocal multinanoplate system under inplane magnetic field", European Journal of Mechanics A/Solids 64, pp. 29 - 45, 2017. DOI: 10.1016/j.euromechsol.2017.01.013

[37] Nematollahi, M. S., Mohammadi, H., Nematollahi, M. A. "Thermal vibration analysis of nanoplates based on the higher-order nonlocal strain gradient theory by an analytical approach", Superlattices and Microstructures 111, pp. 944 - 959, 2017. DOI: 10.1016/j.spmi.2017.07.055

[38] Satish, N., Narendar, S., Brahma Raju, K. "Magnetic field and surface elasticity effects on thermal vibration properties of nanoplates", Composite Structures 180, pp. $568-$ 580, 2017. DOI: $10.1016 /$ j.compstruct.2017.08.028

[39] Shahverdi, H., Barati, M. R. "Vibration analysis of porous functionally graded nanoplates", International Journal of Engineering Science 120, pp. 82 - 99, 2017. DOI: 10.1016/j.ijengsci.2017.06.008

[40] Yang, W. D., Yang, F. P., Wang, X. "Dynamic instability and bifurcation of electrically actuated circular nanoplate considering surface behavior and small scale effect", International Journal of Mechanical Sciences 126, pp. 12 - 23, 2017. DOI: 10.1016/j.ijmecsci.2017.03.018

[41] Zhang, L., Guo, J., Xing, Y. "Bending deformation of multilayered one-dimensional hexagonal piezoelectric quasicrystal nanoplates with nonlocal effect", International Journal of Solids and Structures 132-133, pp. 278-302, 2018. DOI: 10.1016/j.ijsolstr.2017.10.020

[42] Ansari, R., Torabi, J., Norouzzadeh, A. "Bending analysis of embedded nanoplates based on the integral formulation of Eringen's nonlocal theory using the finite element method", Physica B: Condensed Matter 534, pp. 90 - 97, 2018.

[43] Chen, T., Ye, Y., Li, Y. "Investigations on structural intensity in nanoplates with thermal load." Physica E: Low-dimensional Systems and Nanostructures 103, pp. 1 - 9, 2018. DOI: $10.1016 /$ j.physb.2018.01.025

[44] Mohseni, E., Saidi, A. R., Mohammadi, M. "Vibration Analysis of Thick Functionally Graded Micro-plates Using HOSNDPT and Modified Couple Stress Theory", Iranian Journal of Science and Technology, Transactions of Mechanical Engineering 4 (13), pp. 1 -11, 2018. DOI: 10.1007/s40997-018-0185-6 
[45] Karami, B., Janghorban, M., Li, L. "On guided wave propagation in fully clamped porous functionally graded nanoplates", Acta Astronautica 143, pp. 380 - 390, 2018. DOI: $10.1016 /$ j.actaastro.2017.12.011

[46] Yang, L., Li, Y., Gao, Y., Pan, E. "Three-dimensional exact thermo-elastic analysis of multilayered two-dimensional quasicrystal nanoplates", Applied Mathematical Modelling 63, pp. 203 - 218, 2018. DOI: 10.1016/j.apm.2018.06.050

[47] Lin, M.-X., Lee, S.-Y., Chen, C.-K. "Dynamic characteristic analysis of an electrostatically-actuated circular nanoplate subject to surface effects", Applied Mathematical Modelling 63, pp. 18 - 31, 2018. DOI: 10.1016/j.apm.2018.06.004

[48] Pang, M., Li, Z. L., Zhang, Y. Q. "Size-dependent transverse vibration of viscoelastic nanoplates including high-order surface stress effect", Physica B: Condensed Matter 545, pp. 94 - 98, 2018. DOI: 10.1016/j.physb.2018.06.002

[49] Norouzzadeh, A., Ansari, R. "Isogeometric vibration analysis of functionally graded nanoplates with the consideration of nonlocal and surface effects", Thin-Walled Structures 127, pp. 354 - 372, 2018. DOI: 10.1016/j.tws.2017.11.040

[50] Shahrbabaki, E. A. "On three-dimensional nonlocal elasticity: Free vibration of rectangular nanoplate", European Journal of Mechanics - A/Solids 71, pp. 122 - 133, 2018. DOI: $10.1016 /$ j.euromechsol.2018.03.004

[51] Zenkour, A. M. "A novel mixed nonlocal elasticity theory for thermoelastic vibration of nanoplates", Composite Structures 185, pp. $821-833,2018$. DOI: 10.1016/j.compstruct.2017.10.085

[52] Belkorissat, I., Houari, M. S. A., Tounsi, A., Bedia, E. A., Mahmoud, S. R. "On vibration properties of functionally graded nano-plate using a new nonlocal refined four variable model", Steel Compos. Struct 18 (4), pp. 1063 - 1081, 2015. DOI: $10.12989 /$ scs.2015.18.4.1063

[53] Zargaripoor, A., Daneshmehr, A., Hosseini, I. I., Rajabpoor, A. "Free Vibration Analysis of Nanoplates Made of Functionally Graded Materials Based On Nonlocal Elasticity Theory Using Finite Element Method", Journal of Computational Applied Mechanics 49 (1), pp. 86 - 101, 2018. DOI: 10.22059/JCAMECH.2018.248906.223

[54] Eringen, A. C. "Nonlocal continuum field theories", Springer, New York, 2002.

[55] Sobhy, M., Alotebi, M. S. "Transient Hygrothermal Analysis of FG Sandwich Plates Lying on a visco-Pasternak Foundation via a Simple and Accurate Plate Theory." Arabian Journal for Science and Engineering 43 (10), pp. 5423 - 5437, 2018. DOI: $10.1007 / \mathrm{s} 13369-018-3142-1$

[56] Aghababaei, R., Reddy, J. N. "Nonlocal third-order shear deformation plate theory with application to bending and vibration of plates", Journal of Sound and Vibration 326 (1 2), pp. 277 - 289, 2009. DOI: 10.1016/j.jsv.2009.04.044 